\title{
Prognostic Value of Ki-67 Expression in Advanced Lung Squamous Cell Carcinoma Patients Treated with Chemotherapy
}

\author{
Diming Wang $\mathbb{D}^{\prime}$ \\ Wei $\mathrm{Ye}^{2}$ \\ Qingming Shi ${ }^{1}$
}

'Department of Oncology, Anhui Medical University Clinical College of Chest \& Anhui Chest Hospital, Hefei, 230022, People's Republic of China; ${ }^{2}$ Department of Pathology, Anhui Chest Hospital, Hefei, 230022, People's Republic of China
Correspondence: Qingming Shi

Department of Oncology, Anhui Medical University Clinical College of Chest \&

Anhui Chest Hospital, Hefei, 230022,

People's Republic of China

Emailshqm0324@I63.com
Background: The relationship between the Ki-67 expression level and chemotherapy response and survival prognosis in advanced lung squamous cell carcinoma (SCC) remains unclear.

Methods: A total of 101 patients were included in the study. All patients received systemic first-line platinum-based chemotherapy. The Ki-67 expression level was determined by immunohistochemistry analysis.

Results: The Ki-67 expression level was positively correlated with an increase in tumor T stage $(P=0.0140)$, N stage $(P<0.0001)$, and $\mathrm{M}$ stage $(P<0.0001)$ in advanced lung SCC. High Ki-67 expression could predict chemotherapy response (area under the curve $=0.7524$, $P<0.0001)$. Patients with tumors that expressed high levels of Ki-67 had shorter overall survival (OS) (18.8 months vs 25.5 months, $P=0.0002)$ and progression-free survival (PFS) (4.8 months vs 6.7 months, $P<0.0001$ ). Cox analysis found Ki-67 expression to be an independent prognostic biomarker of shortened OS $(P=0.009)$ and PFS $(P=0.008)$.

Conclusion: Ki-67 expression may affect chemotherapy response and thus has prognostic value. Ki-67 expression may be a promising prognostic biomarker for advanced lung SCC. Keywords: Ki-67 expression, advanced lung squamous cell carcinoma, chemotherapy response, prognostic value

\section{Introduction}

Lung cancer is the most common malignancy with the highest incidence and mortality rate worldwide. ${ }^{1}$ Lung squamous cell carcinoma (SCC) represents approximately $30 \%$ of all cases of non-small-cell lung cancer (NSCLC). ${ }^{2}$ Overall, $62 \%$ of lung adenocarcinomas harbor known activating mutations in driver oncogenes, but similar driver mutations are rarely observed in lung SCCs. ${ }^{3,4}$ Programmed death ligand 1 (PD-L1) is a biomarker that has been shown to predict survival outcomes among patients with advanced lung SCC treated with immune checkpoint inhibitors. ${ }^{5}$ Although immunotherapy has changed the treatment pattern of advanced lung SCC, platinum-based doublet chemotherapy is still the main treatment option for most patients, especially for patients with PD-L1 $<1 \%{ }^{6,7}$ Unfortunately, there are no known biomarkers that can predict survival outcomes among patients with advanced lung SCC treated with platinum-based doublet chemotherapy.

$\mathrm{Ki}-67$, a nuclear non-histone protein, has been reported to predict survival outcomes in many cancers, including breast cancer and prostate cancer. ${ }^{8,9}$ Interest in the clinical impact of Ki-67 expression in NSCLCs has recently increased, but its role as a prognostic marker remains unclear. ${ }^{10-13}$ Several studies have suggested that high tumor Ki-67 expression is 
a strong prognostic factor in lung adenocarcinoma. ${ }^{13}$ However, $\mathrm{Ki}-67$ expression is still not considered an established factor for routine use in patients with NSCLC, especially for lung SCCs. ${ }^{13}$ Although a meta-analysis of numerous studies suggested that high $\mathrm{Ki}-67$ expression is a negative prognosticator in lung SCC,${ }^{14}$ the results of this meta-analyses are hampered by a lack of uniformity regarding the chosen cutoff values, confounding the significance of Ki-67 expression in advanced lung SCC. ${ }^{13,14}$ Thus, Ki-67 evaluation in lung SCC has not yet been successfully translated into routine diagnostics.

Therefore, we analyzed the relationship between Ki-67 expression in advanced lung SCC and the clinicopathologic characteristics, chemotherapy responses, and survival prognosis to further understand the significance of Ki-67 expression in advanced lung SCC.

\section{Methods}

\section{Study Population and Design}

We retrospectively analyzed the clinical records of 101 patients with stage IV lung SCC who received systemic first-line platinum-based chemotherapy at the Anhui Chest Hospital between March 2016 and March 2018. Briefly, the screening criteria were as follows: a) stage IV lung SCC, b) 18 to 75 years old, c) Eastern Cooperative Oncology Group (ECOG) performance status score of $0-1$, d) had not received palliative radiotherapy, and e) expected survival time $>3$ months. All patients included in the study had a complete clinical characteristic record and tissue samples available for histologic review and immunohistochemistry analysis. The chemotherapy response was assessed according to the Response Evaluation Criteria in Solid Tumors, version 1.1 (RECIST). All available data were collected, including Ki-67 expression levels and clinicopathological features, including sex, age, ECOG performance status, smoking status, and tumor, node, metastasis (TNM) status. The TNM system of the 7th edition of the International Association for Lung Cancer Research (IASLC) was used for diagnosing and classifying. This study was conducted in accordance with the Declaration of Helsinki and was approved by the Human Research Ethics Committee of Anhui Chest Hospital (no. k2021 - 005). Because of the retrospective nature of the study, the requirement for informed consent was waived.

\section{Immunohistochemistry and Ki-67 Analysis}

Immunohistochemical staining was performed using a Ventana Benchmark XT automated multifunctional slide system (Ventana, Arizona, USA), and a monoclonal antibody against Ki-67 (clone 30-9, Ventana Medical Systems, Inc., USA) was used. The number of tumor cells per sample was calculated by counting and scoring the percentage of positive cells in 5 randomly selected fields at high magnification (400×). The number of Ki-67-positive tumor cells per 100 tumor cells was counted in each microscopic field. The percentage of positive tumor cells was scored to assess $\mathrm{Ki}$ 67 immunostaining as follows: positive if brownish-yellow staining was seen in the nucleus and Ki-67-positive cells were $>5 \%$; negative if no brownish-yellow staining was observed or Ki-67-positive cells were $<5 \%$. Next, Ki-67 expression was scored according to the percentage of positive cells: 1 for $<15 \%, 2$ for $16 \%-45 \%, 3$ for $46 \%-75 \%$, and 4 for $>75 \%$ (Figure 1).

\section{Statistical Analysis}

The statistical analysis was performed using GraphPad Prism 7.0 (GraphPad Software, San Diego, CA, USA) or SPSS 16.0 (IBM SPSS, Chicago, IL, USA). Continuous variables are presented as the mean $\pm \mathrm{SEM}$, while numerical variables are given as $\mathrm{N}(\%)$. The relationship between the clinicopathological features of patients with advanced lung SCC and Ki-67 expression was assessed by Pearson's chi-squared test. The relationship between Ki-67 expression and chemotherapy response was assessed by unpaired $t$-test. The predictive value of $\mathrm{Ki}-67$ expression in determining the objective response rate (ORR) of advanced lung SCC was assessed by receiver operator characteristic (ROC) curve analysis. The correlations between Ki-67 expression level and the progression-free survival (PFS) and overall survival (OS) rates were determined by the Kaplan-Meier curve and Log rank test. The Cox proportional hazards regression model was used to assess risk factors. P-values $<0.05$ were considered statistically significant.

\section{Results}

\section{Baseline Characteristics}

All enrolled patients had pathologically confirmed stage IV (M1a: 75.2\%; M1b: 24.8\%) lung SCC. The mean Ki-67 expression level was $51.53 \pm 2.231 \%$ across all samples. The mean patient age was $63.46 \pm 0.772$ years. Sixty-eight patients were male $(67.3 \%)$, and 33 were female $(32.7 \%)$. Seventy-five $(74.3 \%)$ patients were current or former smokers, and $26(25.7 \%)$ patients were never smokers (Table 1). 

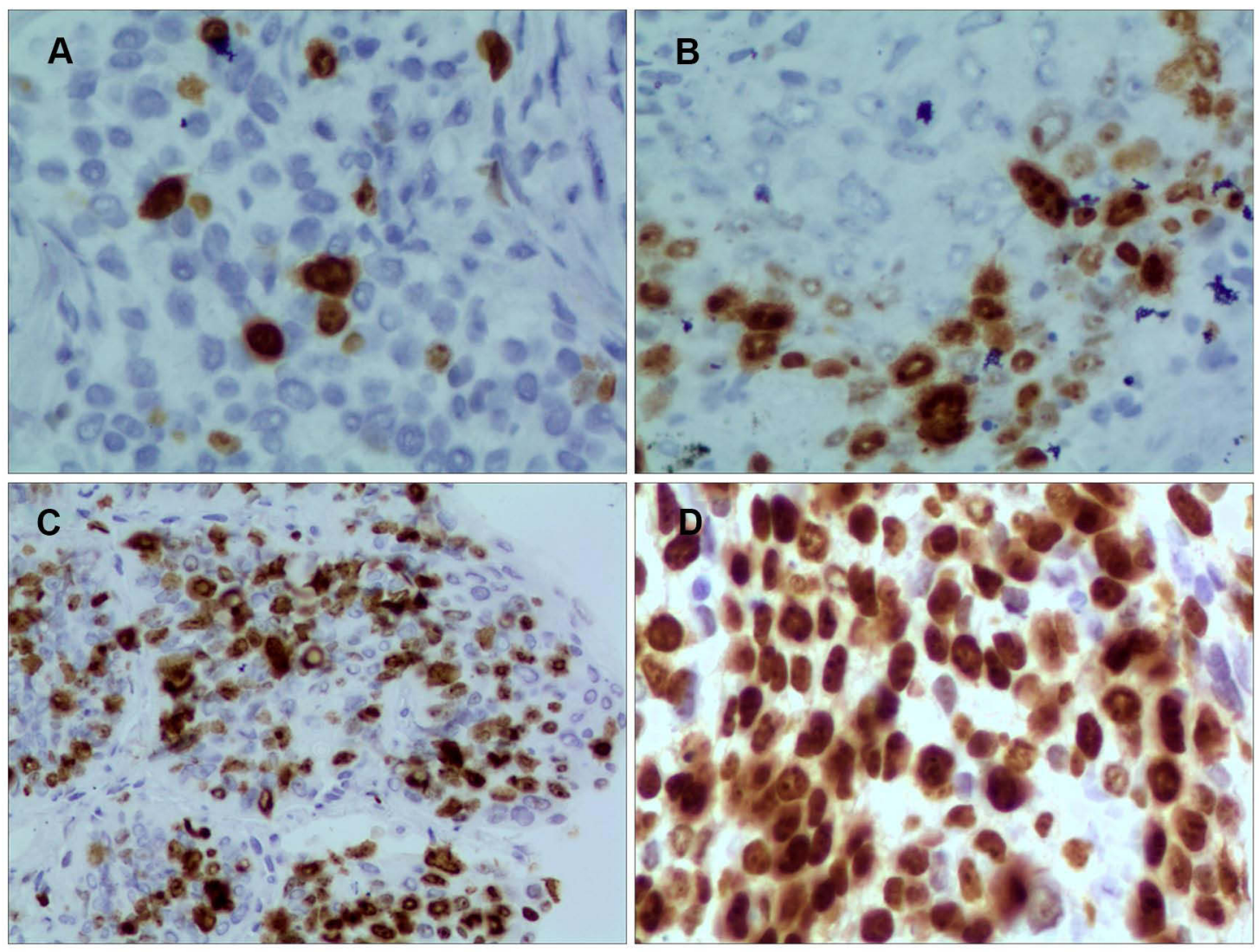

Figure I Immunohistochemical staining of Ki-67 expression in advanced lung SCC. (magnification 400x). (A) Score I: low; (B) score 2: relatively low; (C) score 3: high, and (D) score 4: very high.

Abbreviation: SCC, squamous cell carcinoma.

\section{Correlation Between Ki-67 Expression and the Clinicopathological Parameters of Advanced Lung SCC}

Based on the differences in the Ki-67 expression level, we analyzed the relationship between Ki-67 expression and the clinicopathological characteristics of patients with advanced lung SCC. The expression of Ki-67 increased with increasing tumor T stage $(P=0.0140), \mathrm{N}$ stage $(P<0.0001)$, and $\mathrm{M}$ stage $(P<0.0001)$ (Figure $2 \mathrm{E}-\mathrm{G})$. However, there was no significant association between $\mathrm{Ki}-67$ expression and the other clinicopathological features analyzed (Figure 2A-D).

\section{Association Between High Ki-67 Expression and Chemotherapy Response in Patients with Advanced Lung SCC}

All patients were included in the evaluation of treatment efficacy after 2 courses of chemotherapy. No patients achieved a complete response, 44 patients achieved a partial response, 50 patients had stable disease, and 7 patients experienced progressive disease. The ORR was $43.6 \%$, and the disease control rate (DCR) was $93.1 \%$. We found that high Ki-67 expression was associated with ORR $(P<0.0001)$ but not with DCR $(P=0.2182)$ in patients with advanced lung SCC (Figure 3A and B). We analyzed the ROC curve for the ORR to more accurately determine the significance of Ki-67 expression in the chemotherapy response of patients with advanced lung SCC. Our results suggested that high Ki-67 expression could be a promising biomarker for the ORR of patients with advanced lung SCC. The area under the curve (AUC) was 0.7524 [95\% confidence interval (CI): 0.6568-0.8479], with a sensitivity of 0.6316 (95\% CI: $0.4934-0.7555)$ and a specificity of 0.7727 (95\% CI: $0.6216-0.8853)$ when the cutoff value was set at $45 \%$ according to our detection method (Figure 3C).

\section{High Ki-67 Expression Levels Indicate a Poor Clinical Prognosis}

Based on the cutoff value of the Ki-67 level, the patients were divided into a high $\mathrm{Ki}-67$ expression group and a low 
Table I Tumor Characteristics of Patients with Advanced Lung SCC

\begin{tabular}{|c|c|}
\hline Characteristic & $\mathbf{N}(\%)$ \\
\hline \multicolumn{2}{|l|}{ Age } \\
\hline Mean \pm SEM, years & $63.46 \pm 0.772$ \\
\hline \multicolumn{2}{|l|}{ Sex } \\
\hline Male/female & $68 / 33$ \\
\hline \multicolumn{2}{|l|}{ ECOG performance status } \\
\hline $0 / 1$ & $22 / 79$ \\
\hline \multicolumn{2}{|l|}{ Smoking status } \\
\hline Never smoker/ current or former smoker & $26 / 75$ \\
\hline \multicolumn{2}{|l|}{$\mathrm{Ki}-67$} \\
\hline Mean \pm SEM, (\%) & $51.53 \pm 2.231$ \\
\hline \multicolumn{2}{|l|}{ T stage } \\
\hline $\mathrm{TI}$ & $0(0.0)$ \\
\hline $\mathrm{T} 2$ & $44(43.6)$ \\
\hline T3 & $35(34.6)$ \\
\hline $\mathrm{T} 4$ & $22(21.8)$ \\
\hline \multicolumn{2}{|l|}{$\mathrm{N}$ stage } \\
\hline No & $0(0.0)$ \\
\hline NI & $48(47.5)$ \\
\hline N2 & $42(4 I .6)$ \\
\hline N3 & II (I0.9) \\
\hline \multicolumn{2}{|l|}{ M stage } \\
\hline Mla & $76(75.2)$ \\
\hline MIb & $25(24.8)$ \\
\hline
\end{tabular}

Abbreviations: SCC, squamous cell carcinoma; ECOG, eastern cooperative oncology group; T, tumor; N, node; M, metastasis; SEM, standard error of the mean.

Ki-67 expression group. We further analyzed the correlation between the OS and PFS rates and the Ki-67 expression levels in patients with advanced lung SCC (Figure 4A and $\mathrm{B})$. We determined the OS rates in a group of 101 patients and found that there was a significant difference in the OS rate between the high $\mathrm{Ki}-67$ expression group and the low Ki-67 expression group (OS: 18.8 months vs 25.5 months, respectively, $P=0.0002)$. As expected, an analysis of the PFS yielded similar results (PFS: 4.8 months vs 6.7 months in the high expression and low expression groups, respectively, $P<0.0001)$. These results indicated that high expression of Ki-67 predicted a poor outcome in patients with advanced lung SCC. Thereafter, we analyzed all clinicopathological factors that influenced the PFS and OS rates by univariate and multivariate Cox regression analysis (Tables 2 and 3). The multivariate analysis identified Ki-67 expression as an independent factor that influenced the PFS $(P=0.008)$ and OS rates $(P=0.009)$.

\section{Discussion}

Ki-67 is a DNA-binding nuclear non-histone protein first identified 30 years ago. ${ }^{15}$ However, the role of Ki-67 and its value as a biomarker in lung cancer have not previously been determined. In this study, we analyzed the relationship between Ki-67 expression and the clinical features of stage IV lung SCC patients treated with chemotherapy. We found that the $\mathrm{T}$ stage, $\mathrm{N}$ stage, and distant metastasis all had a strong link with high Ki-67 expression. We also evaluated the clinical value of the Ki-67 expression level to predict the chemotherapy response and prognosis of patients with advanced lung SCC. ROC curve analysis showed that Ki-67 could be used as a biomarker for predicting chemotherapy response. We also found that Ki-67 expression was a poor prognostic indicator for OS and PFS in patients with stage IV lung SCC. We concluded from the Cox regression analysis that Ki-67 expression might serve as an independent prognostic biomarker in advanced lung SCC.

In a previous study, Warth et al ${ }^{16}$ showed that Ki-67 expression was not significantly associated with $\mathrm{T}$ stage $(P=0.374), \mathrm{N}$ stage $(P=0.807)$, or M stage $(P=0.527)$ in lung SCC. In addition, Masuda et $\mathrm{a}^{17}$ suggested that there was no significant correlation between Ki-67 expression and T stage $(P=0.186)$ or $\mathrm{N}$ stage $(P=0.539)$. However, previous clinical studies primarily included patients with stage I-II disease and few patients with stage IV disease. ${ }^{16-21}$ Our study enrolled patients with stage IV lung SCC and found that Ki-67 expression was significantly associated with $\mathrm{T}, \mathrm{N}$, and $\mathrm{M}$ stage.

Although much effort has been exerted to explore biomarkers that can predict the efficacy of chemotherapy, as yet no biomarkers have been found to accurately predict chemotherapy efficacy in lung SCC. ${ }^{22}$ Warth et al ${ }^{16}$ showed that Ki-67 expression positively impacted the survival of patients with lung SCC, but Ki-67 expression failed to predict a benefit from adjuvant chemotherapy in that study. Masuda et $\mathrm{al}^{17}$ showed that high Ki-67 expression levels were a significant indicator of poor prognosis in patients with lung SCC. However, the study only demonstrated that Ki67 expression was associated with tumor infiltration patterns. Hence, it is unclear from these previous studies whether Ki67 expression can predict chemotherapy efficacy. ${ }^{16-21}$ The current study is the first to identify that high Ki-67 expression can accurately predict chemotherapy efficacy in advanced lung SCC. We found an improved chemotherapy efficacy in patients with high Ki-67 expression; however, OS and PFS 
A

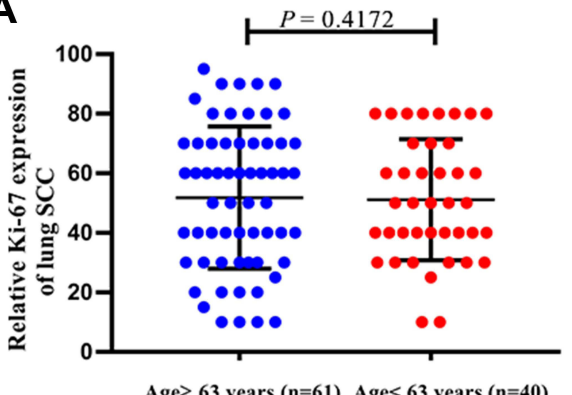

C

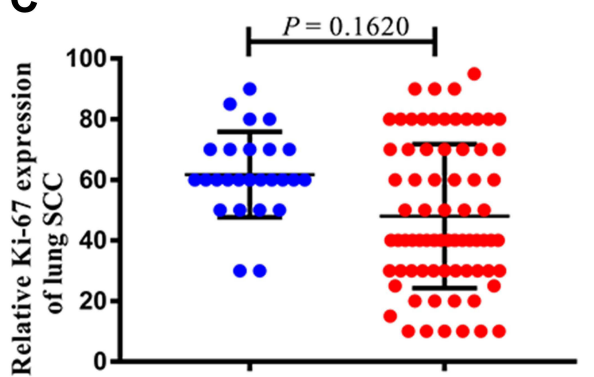

Never smoker $(n=26)$ Current or former smoker $(n=75)$
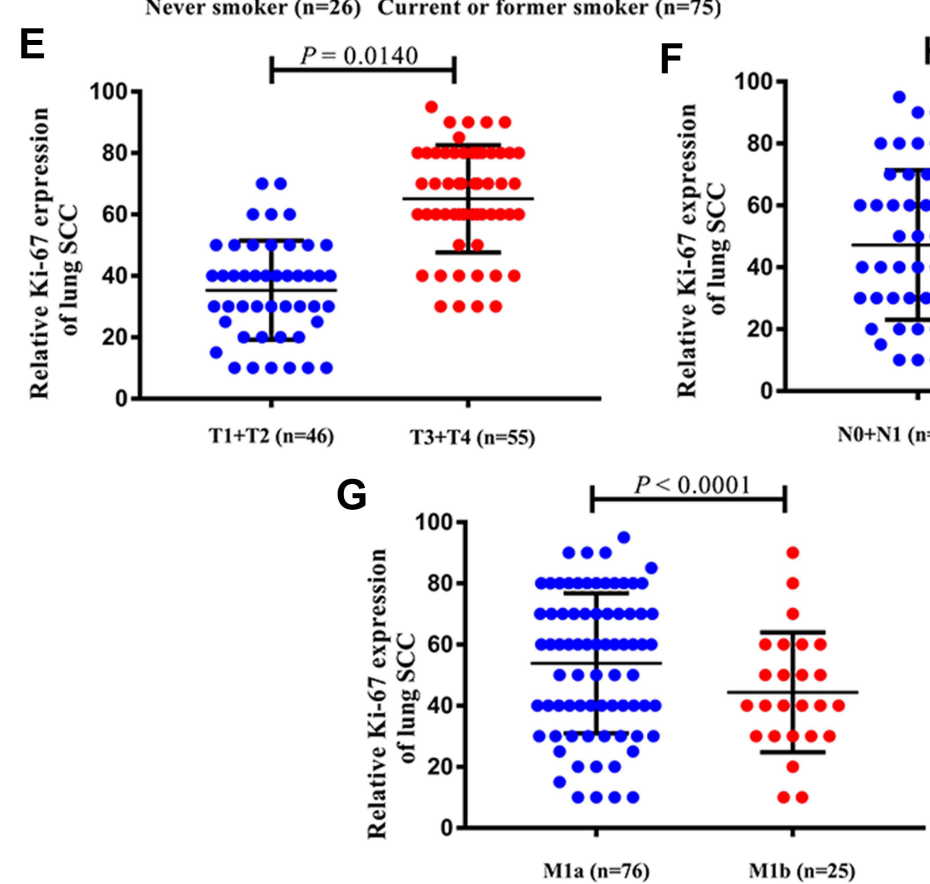

B

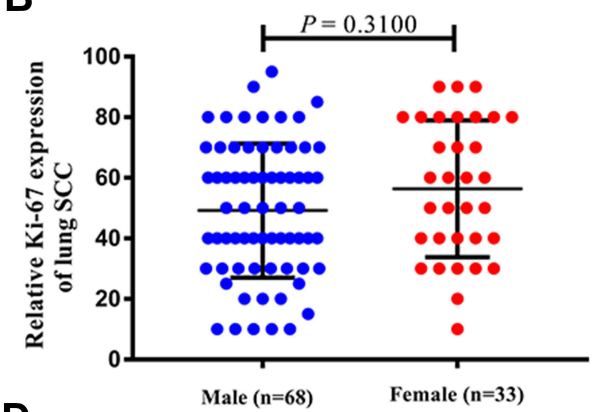

D
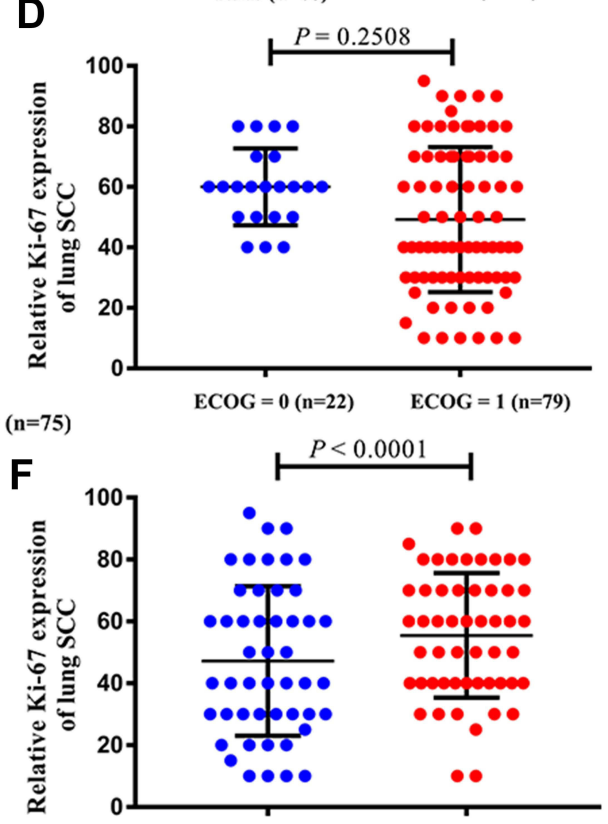

$\mathrm{N} 2+\mathrm{N} 3(\mathrm{n}=53)$

Figure 2 Relative expression of Ki-67 in subgroups: (A) age, (B) sex, (C) smoking status, (D) ECOG PS, (E) T stage, (F) N stage, and (G) M stage. Abbreviations: ECOG PS, eastern cooperative oncology group performance status; T, tumor; N, node; M, metastasis.

were not satisfactory. Therefore, it is important to explore ways to improve the prognosis of patients with advanced lung SCC who have high Ki-67 expression. Immune checkpoint inhibitors have changed the therapeutic approach to advanced lung SCC. ${ }^{23}$ Immunotherapy combined with platinum-based doublet chemotherapy can provide better outcomes than single platinum-based doublet chemotherapy. ${ }^{24}$ Several studies have demonstrated a correlation between $\mathrm{Ki}-67$ expression and PD-L1 in NSCLC. ${ }^{19,25}$ Hence, we will explore the correlation between $\mathrm{Ki}-67$ expression and $\mathrm{PD}-\mathrm{L} 1$ expression to further enhance the survival prognosis of advanced lung SCC in the future.

There are some limitations to this study. Owing to the retrospective study design, only patients with stage IV lung SCC were included and no control group was established, such as Ki-67 expression in healthy tissue or Ki-67 

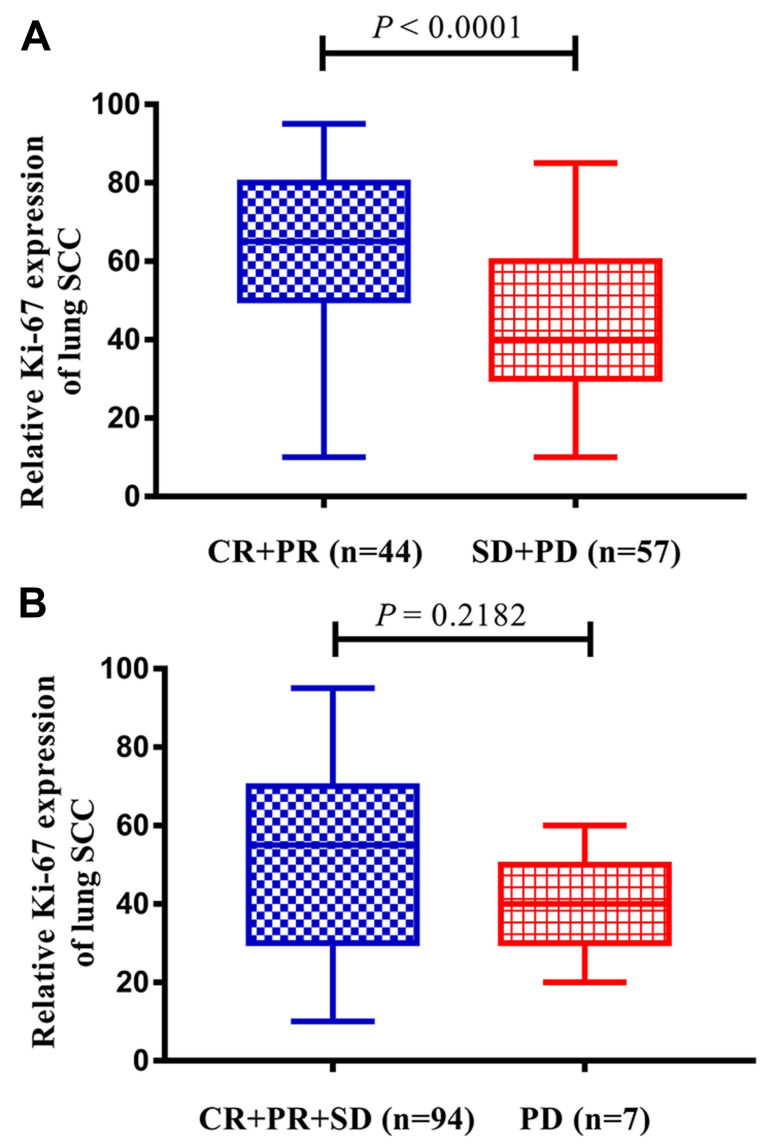

C

\section{CR+PR vs. SD+PD}

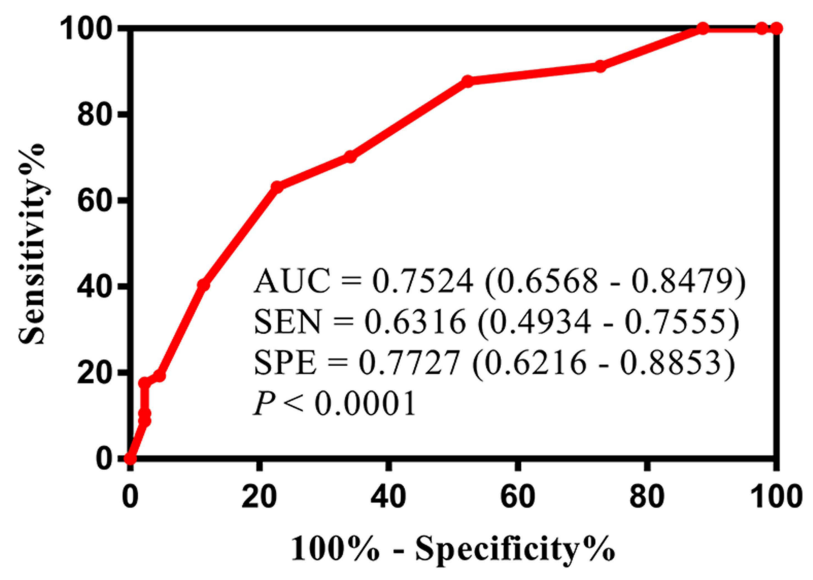

Figure 3 High Ki-67 expression can be considered a biomarker for the objective efficacy of chemotherapy in advanced lung squamous cell carcinoma (SCC). (A) Comparison between $\mathrm{Ki}-67$ expression in the $\mathrm{CR}+\mathrm{PR}$ group and SD+PD group. (B) Comparison between $\mathrm{Ki}-67$ expression in the $\mathrm{CR}+\mathrm{PR}+\mathrm{SD}$ group and $\mathrm{PD}$ group. (C) The expression levels of $\mathrm{Ki}-67$ could distinguish patients with high objective efficacy from patients with low objective efficacy in advanced lung SCC according to the ROC analysis.

Abbreviations: CR, complete response; PR, partial response; SD, stable disease; $\mathrm{PD}$, progressive disease; ROC, receiver operator characteristic; AUC, area under the curve.

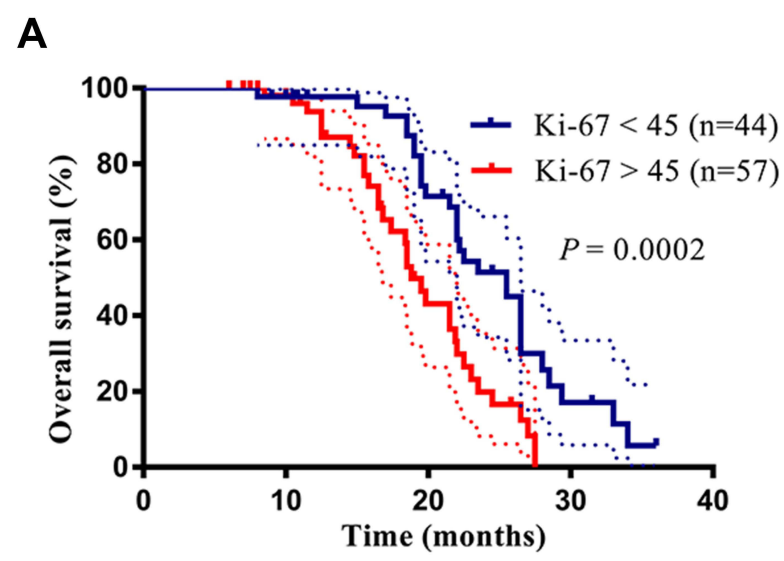

B

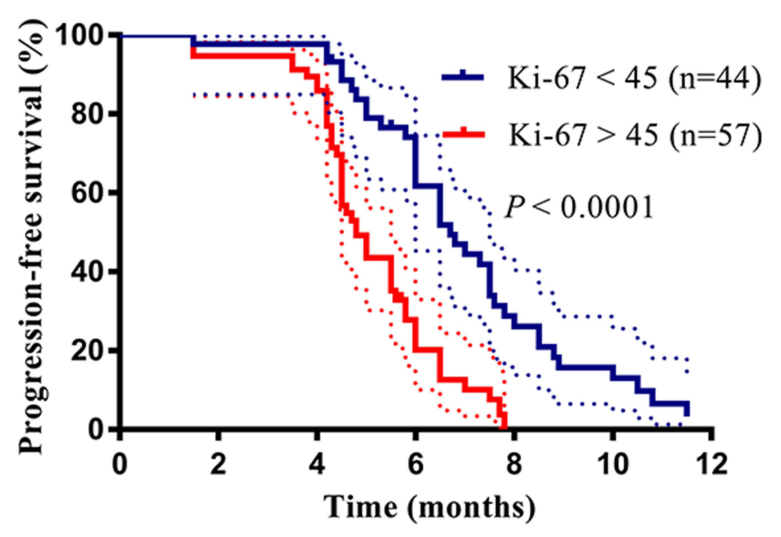

Figure 4 High expression level of Ki-67 predicts a poor outcome for advanced lung squamous cell carcinoma (SCC) patients. (A) Patients with high $\mathrm{Ki}-67$ expression levels have a shortened overall survival (OS). (B) Patients with high Ki-67 expression levels have a shortened progression-free survival (PFS).

expression in early-stage tumor tissue. In addition, the sample size is relatively small. These may have led to bias in the results. We plan to conduct a series of prospective studies that will enroll different cohorts to further investigate the significance of $\mathrm{Ki}-67$ expression in lung SCC.

\section{Conclusions}

Our findings confirm that Ki-67 expression plays an important role in predicting chemotherapy efficacy and determining survival prognosis. Ki-67, as a promising prognostic biomarker for advanced lung SCC, should be further investigated. 
Table 2 Cox Regression Analysis of Prognostic Risk Factors and Patient Progression-Free Survival

\begin{tabular}{|c|c|c|c|c|c|c|}
\hline \multirow[t]{2}{*}{ Risk Factors } & \multicolumn{3}{|c|}{ Univariate Analysis } & \multicolumn{3}{|c|}{ Multivariate Analysis } \\
\hline & HR & $95 \% \mathrm{Cl}$ & $P$ value & HR & $95 \% \mathrm{Cl}$ & $P$ value \\
\hline Sex & 1.745 & I.108-2.749 & $0.016^{*}$ & 1.062 & $0.556-2.026$ & 0.856 \\
\hline Age & 1.018 & $0.989-1.047$ & 0.233 & & & \\
\hline ECOG PS & 0.754 & $0.437-1.300$ & 0.310 & & & \\
\hline Smoking status & 0.706 & $0.427-1.166$ & 0.174 & & & \\
\hline T stage & 2.247 & $\mathrm{I} .65 \mathrm{I}-3.058$ & $0.000^{*}$ & 1.448 & $0.90 \mathrm{I}-2.329$ & 0.126 \\
\hline $\mathrm{N}$ stage & 1.740 & I.242-2.437 & $0.00 I^{*}$ & 1.253 & $0.767-2.045$ & 0.367 \\
\hline M stage & 1.064 & $0.648-1.745$ & 0.807 & & & \\
\hline Ki-67 & 1.027 & $1.016-1.037$ & $0.000 *$ & 1.019 & $1.005-1.033$ & $0.008^{*}$ \\
\hline
\end{tabular}

Note: $* p<0.05$.

Abbreviations: T, tumor; N, node; M, metastasis; ECOG PS, eastern cooperative oncology group performance status; HR, hazard ratio; Cl, confidence interval.

Table 3 Cox Regression Analysis of Prognostic Risk Factors and Patient Overall Survival

\begin{tabular}{|l|l|l|l|l|l|l|}
\hline \multirow{2}{*}{ Risk Factors } & \multicolumn{2}{l|}{ Univariate Analysis } & \multicolumn{2}{l|}{ Multivariate Analysis } \\
\cline { 2 - 6 } & HR & $\mathbf{9 5 \%} \mathbf{C l}$ & $\mathbf{P}$ value & HR & $\mathbf{9 5 \%} \mathbf{C l}$ \\
\hline Sex & 1.991 & $1.161-3.412$ & $0.012^{*}$ & 1.288 & $0.598-2.775$ \\
Age & 1.033 & $0.999-1.068$ & 0.059 & & \\
ECOG PS & 1.240 & $0.641-2.401$ & 0.523 & & \\
Smoking status & 0.893 & $0.496-1.606$ & 0.705 & & \\
T stage & 1.804 & $1.328-2.451$ & $0.000^{*}$ & 1.116 & $0.683-1.825$ \\
N stage & 1.849 & $1.213-2.819$ & $0.004^{*}$ & 1.216 & $0.645-2.294$ \\
M stage & 1.100 & $0.621-1.948$ & 0.744 & & & 0.662 \\
Ki-67 & 1.029 & $1.015-1.044$ & $0.000^{*}$ & 1.024 & $1.006-1.043$ \\
\hline
\end{tabular}

Note: $* p<0.05$.

Abbreviations: T, tumor; N, node; M, metastasis; ECOG PS, eastern cooperative oncology group performance status; HR, hazard ratio; Cl, confidence interval.

\section{Ethical Approval}

This study was conducted in accordance with the Declaration of Helsinki and was approved by the Human Research Ethics Committee of Anhui Chest Hospital (no. k2021 - 005). The reasons for the waiver were as follows. First, the study was a retrospective analysis of patients' previous diagnosis and treatment data; thus, there was no additional risk to patients. Additionally, the ethics committee waived the requirement for informed written consent. All data were anonymized to maintain patient privacy.

\section{Disclosure}

The authors have no conflicts of interest to declare.

\section{References}

1. Siegel RL, Miller KD, Jemal A. Cancer statistics, 2020. CA Cancer J Clin. 2020;70(1):7-30. doi:10.3322/caac.21590

2. Travis WD. Lung cancer pathology: current concepts. Clin Chest Med. 2020;41(1):67-85. doi:10.1016/j.ccm.2019.11.001
3. Zhang XC, Wang J, Shao GG, et al. Comprehensive genomic and immunological characterization of Chinese non-small cell lung cancer patients. Nat Commun. 2019;10(1):1772. doi:10.1038/s41467-019-09762-1

4. Chang YS, Tu SJ, Chen YC, et al. Mutation profile of non-small cell lung cancer revealed by next generation sequencing. Respir Res. 2021;22(1):3. doi:10.1186/s12931-020-01608-5

5. Paz-Ares L, Luft A, Vicente D, et al. Pembrolizumab plus chemotherapy for squamous non-small-cell lung cancer. $N$ Engl $J$ Med. 2018;379(21):2040-2051. doi:10.1056/NEJMoa1810865

6. Ang YL, Tan HL, Soo RA. Best practice in the treatment of advanced squamous cell lung cancer. Ther Adv Respir Dis. 2015;9(5):224-235. doi:10.1177/1753465815581147

7. Hirsch FR, Kerr KM, Bunn PA Jr., et al. Molecular and immune biomarker testing in squamous-cell lung cancer: effect of current and future therapies and technologies. Clin Lung Cancer. 2018;19 (4):331-339. doi:10.1016/j.cllc.2018.03.014

8. Zhu X, Chen L, Huang B, et al. The prognostic and predictive potential of Ki-67 in triple-negative breast cancer. Sci Rep. 2020;10 (1):225. doi:10.1038/s41598-019-57094-3

9. Kammerer-Jacquet SF, Ahmad A, Møller H, et al. Ki-67 is an independent predictor of prostate cancer death in routine needle biopsy samples: proving utility for routine assessments. Mod Pathol. 2019;32(9):1303-1309. doi:10.1038/s41379-019-0268-y

10. Lin L, Cheng J, Tang D, et al. The associations among quantitative spectral CT parameters, Ki-67 expression levels and EGFR mutation status in NSCLC. Sci Rep. 2020;10(1):3436. doi:10.1038/s41598020-60445-0 
11. Xu J, Liu P, Da J, Hao J, Peng W, Sun G. Prognostic value of Ki-67 in stage I non-small-cell lung cancer: a meta-analysis involving 1931 patients. Pathol Res Pract. 2019;215(5):855-860. doi:10.1016/j. prp.2019.02.020

12. Mitchell KG, Parra ER, Nelson DB, et al. Tumor cellular proliferation is associated with enhanced immune checkpoint expression in stage I non-small cell lung cancer. $J$ Thorac Cardiovasc Surg. 2019;158(3):911-919.e6. doi:10.1016/j.jtcvs.2019.04.084

13. Wen $\mathrm{S}$, Zhou W, Li CM, et al. Ki-67 as a prognostic marker in early-stage non-small cell lung cancer in Asian patients: a meta-analysis of published studies involving 32 studies. BMC Cancer. 2015;15:520. doi:10.1186/s12885-015-1524-2

14. Martin B, Paesmans M, Mascaux C, et al. Ki-67 expression and patients survival in lung cancer: systematic review of the literature with meta-analysis. $B r \quad J$ Cancer. 2004;91(12):2018-2025. doi:10.1038/sj.bjc.6602233

15. Menon SS, Guruvayoorappan C, Sakthivel KM, Rasmi RR. Ki-67 protein as a tumour proliferation marker. Clin Chim Acta. 2019;491:39-45. doi:10.1016/j.cca.2019.01.011

16. Warth A, Cortis J, Soltermann A, et al. Tumour cell proliferation (Ki-67) in non-small cell lung cancer: a critical reappraisal of its prognostic role. Br J Cancer. 2014;111(6):1222-1229. doi:10.1038/ bjc. 2014.402

17. Masuda D, Masuda R, Matsuzaki T, et al. Ki-67 labeling index affects tumor infiltration patterns of lung squamous cell carcinoma. Mol Med Rep. 2015;12(5):7303-7309. doi:10.3892/mmr.2015.4354

18. Zhu WY, Hu XF, Fang KX, et al. Prognostic value of mutant p53, Ki-67, and TTF-1 and their correlation with EGFR mutation in patients with non-small cell lung cancer. Histol Histopathol. 2019;34(11):1269-1278.
19. Pezzuto A, Cappuzzo F, D’Arcangelo M, et al. Prognostic value of p16 protein in patients with surgically treated non-small cell lung cancer; relationship with Ki-67 and PD-L1. Anticancer Res. 2020;40 (2):983-990. doi:10.21873/anticanres.14032

20. Grant L, Banerji S, Murphy L, et al. Androgen receptor and Ki67 expression and survival outcomes in non-small cell lung cancer. Horm Cancer. 2018;9(4):288-294. doi:10.1007/s12672-018-0336-7

21. Jakobsen JN, Sørensen JB. Clinical impact of ki-67 labeling index in non-small cell lung cancer. Lung Cancer. 2013;79(1):1-7. doi:10.1016/j.lungcan.2012.10.008

22. Thakur MK, Gadgeel SM. Predictive and prognostic biomarkers in non-small cell lung cancer. Semin Respir Crit Care Med. 2016;37 (5):760-770. doi:10.1055/s-0036-1592337

23. Chen RL, Zhou JX, Cao Y, et al. The efficacy of PD-1/PD-L1 inhibitors in advanced squamous-cell lung cancer: a meta-analysis of 3112 patients. Immunotherapy. 2019;11(17):1481-1490. doi:10.2217/imt-2019-0101

24. Mok TSK, Wu YL, Kudaba I, et al. Pembrolizumab versus chemotherapy for previously untreated, PD-L1-expressing, locally advanced or metastatic non-small-cell lung cancer (KEYNOTE-042): a randomised, open-label, controlled, Phase 3 trial. Lancet. 2019;393(10183):1819-1830. doi:10.1016/S01406736(18)32409-7

25. Pawelczyk K, Piotrowska A, Ciesielska U, et al. Role of PD-L1 expression in non-small cell lung cancer and their prognostic significance according to clinicopathological factors and diagnostic markers. Int J Mol Sci. 2019;20(4):824. doi:10.3390/ijms20040824

\section{Publish your work in this journal}

Cancer Management and Research is an international, peer-reviewed open access journal focusing on cancer research and the optimal use of preventative and integrated treatment interventions to achieve improved outcomes, enhanced survival and quality of life for the cancer patient.
The manuscript management system is completely online and includes a very quick and fair peer-review system, which is all easy to use. Visit http://www.dovepress.com/testimonials.php to read real quotes from published authors. 